University of Texas at El Paso

ScholarWorks@UTEP

4-2007

\title{
Von Mises Failure Criterion in Mechanics of Materials: How to Efficiently Use It Under Interval and Fuzzy Uncertainty
}

\author{
Gang Xiang \\ Andrzej Pownuk \\ The University of Texas at El Paso, ampownuk@utep.edu \\ Olga Kosheleva \\ The University of Texas at El Paso, olgak@utep.edu \\ Scott A. Starks \\ The University of Texas at El Paso, sstarks@utep.edu
}

Follow this and additional works at: https://scholarworks.utep.edu/cs_techrep

Part of the Computer Engineering Commons

Comments:

Technical Report: UTEP-CS-07-24

Published in: Marek Reformat and Michael R. Berthold (eds.), Proceedings of the 26th International Conference of the North American Fuzzy Information Processing Society NAFIPS'2007, San Diego, California, June 24-27, 2007, pp. 570-575.

\section{Recommended Citation}

Xiang, Gang; Pownuk, Andrzej; Kosheleva, Olga; and Starks, Scott A., "Von Mises Failure Criterion in Mechanics of Materials: How to Efficiently Use It Under Interval and Fuzzy Uncertainty" (2007). Departmental Technical Reports (CS). 145. https://scholarworks.utep.edu/cs_techrep/145

This Article is brought to you for free and open access by the Computer Science at ScholarWorks@UTEP. It has been accepted for inclusion in Departmental Technical Reports (CS) by an authorized administrator of ScholarWorks@UTEP. For more information, please contact Iweber@utep.edu. 


\title{
Von Mises Failure Criterion in Mechanics of Materials: How to Efficiently Use it Under Interval and Fuzzy Uncertainty
}

\author{
Gang Xiang*, Andrzej Pownuk ${ }^{\dagger}$ \\ Olga Kosheleva ${ }^{\ddagger}$, and Scott A. Starks ${ }^{\S}$ \\ *Department of Computer Science \\ ${ }^{\dagger}$ Department of Mathematical Sciences \\ ‡Department of Teacher Education \\ $\S$ Department of Electrical and Computer Engineering \\ University of Texas at El Paso \\ El Paso, TX 79968, USA \\ *gxiang@utep.edu, †ampownuk@utep.edu, \\ †olgak@utep.edu, *sstarks@utep.edu
}

\begin{abstract}
One of the main objective of mechanics of materials is to predict when the material experiences fracture (fails), and to prevent this failure. With this objective in mind, it is desirable to use it ductile materials, i.e., materials which can sustain large deformations without failure. Von Mises criterion enables us to predict the failure of such ductile materials. To apply this criterion, we need to know the exact stresses applied at different directions. In practice, we only know these stresses with interval or fuzzy uncertainty. In this paper, we describe how we can apply this criterion under such uncertainty, and how to make this application computationally efficient.
\end{abstract}

\section{What is Von Mises CRITERION: IN BRIEF}

\section{A. Basics of Mechanics of Materials: the Notion of Stress}

When a force is applied to a material, this material deforms and at some point breaks down. We can gauge the effect of the force by the stress, the force per unit area. The larger the stress, the larger the deformation; at some point, larger stress leads to a breakdown.

\section{B. Case of Small Stress: Elastic (Reversible) Deformations}

When the stress is small, no irreversible damage occurs, all deformations are reversible.

The original shape of the material (i.e., the shape in the absence of stress) is the one to which the undamaged material reverts. Thus, under small stress, the material returns to its original shape once the force is no longer applied. Such reversible deformation which return to the original shape is called elastic.

\section{Case of Larger Stress: Irreversible (Plastic) Deformation}

An increased level of stress causes irreversible damage in the material. In this case, after the force is no longer applied, the material does not return to its original shape. Such irreversible deformation is called plastic or yielding.

\section{From Plastic Deformation to Failure}

Under plastic deformation, there is an irreversible damage to the material, but this damage occurs on the microlevel. On the macrolevel, the material may be slightly misshapen and somewhat twisted, but it is still intact and it can still serve its purpose.

However, as the stress increases, it causes macrodamage too: the material experiences fractures. In many mechanical designs, the fractured material can no longer fulfil its duties, so it is usually said that this material fails.

\section{E. Predicting Failure is Extremely Important}

Material failure can have catastrophic consequences. As a result, it is extremely important to predict when a material can fail.

In many practical situations, it is also important to know when the yielding starts, because while the yielding itself is usually not catastrophic, the resulting irreversible damage start weakening the mechanical construction can lead to a failure in the long run.

\section{F. Case of 1-D Stress}

Let us first consider the simplest situation of a 1-D stress, when the force is only applied in one direction. In this case, as we have mentioned, both the yielding and the failure start when the stress becomes large enough. In other words, for 1-D stress, for each material, there are two thresholds:

- the threshold $\sigma_{y}$ after which yielding starts, and

- the threshold $\sigma_{f}>\sigma_{y}$ after which the material fails.

\section{G. Ductile Materials and their Practical Importance}

In practical applications, it is desirable to use materials which can sustain large deformations without failure.

This is not always possible: e.g., some materials such as ceramics fail almost immediately after the yielding starts. 
However, many other materials can sustain large plastic deformations without failure. Such materials are called ductile. Examples of ductile materials include ductile metals such as copper, silver, gold, and steel; it is possible to deform these ductile metals into wire without breaking them.

In view of many important applications of ductile materials, it is necessary to predict when they fail.

\section{H. Case of General (3-D) Stress: Importance}

We have mentioned that for 1-D stress, it is easy to predict when a material fails: when the stress exceeds its failure threshold.

In real life, situations in which the force comes from only one direction are rare. Usually, have a combination of stresses coming from different directions. It is therefore important to be able to predict when a material fails under such 3-D stress.

\section{Case of General (3-D) Stress: Formulation of the Problem}

A general 3-D stress can be described as a combination of three stresses $\sigma_{1}, \sigma_{2}$, and $\sigma_{3}$ applied at three orthogonal directions. It is therefore desirable to be able, given the three stresses $\sigma_{i}$, to be able to predict when a ductile material fails under these stresses.

\section{J. Maxwell's Mathematical Solution to the Problem}

First solution to this problem was provided by Maxwell. The first solution to this important problem was provided by Maxwell (of the electromagnetic equations fame) in the 1860s. As we will see, Maxwell's formulas are still used to predict the material's failure.

Because of the continuing practical importance of Maxwell's solution, in this section, we will briefly reproduce Maxwell's derivations - to make the resulting formulas more understandable. (Of course, our rendering of Maxwell's derivation will be somewhat modernized.)

Those readers who are already familiar with failure mechanics and with the von Mises criterion (and with its motivations) are welcome skip this section.

Need for an appropriate combination of stresses: physical motivations. In the 1-D case, the corresponding stress $\sigma$ provides a numerical measure of how stressed the material is: when this stress exceeds a given threshold $\sigma_{f}$, the material fails.

In the 3-D case, we have three different stresses $\sigma_{1}, \sigma_{2}$, and $\sigma_{3}$. Informally, all these three stresses contribute to the "overall stress". When this "combined stress" exceeds a certain threshold, the material fails. Thus, to be able to predict when a material fails, we must be able to find out how this "combined stress" depends on the individual stresses $\sigma_{i}$.

Maxwell's main idea is thus to combine the there stresses $\sigma_{1}, \sigma_{2}$, and $\sigma_{3}$ into a single numerical criterion $f\left(\sigma_{1}, \sigma_{2}, \sigma_{3}\right)$ that would decide when a material fails. To be more precise, Maxwell assumed that there exists a threshold value $f_{0}$ such that:

- when $f\left(\sigma_{1}, \sigma_{2}, \sigma_{3}\right)<f_{0}$, the material remains intact (i.e., undamaged on the macro level);
- when $f\left(\sigma_{1}, \sigma_{2}, \sigma_{3}\right) \geq f_{0}$, the material fails.

Need for an appropriate combination of stresses: mathematical motivations. The existence of a combination function $f\left(\sigma_{1}, \sigma_{2}, \sigma_{3}\right)$ is motivated not only by physics, it can also be justified on purely mathematical grounds.

Specifically, for every material and for every triple $\left(\sigma_{1}, \sigma_{2}, \sigma_{3}\right)$ of the corresponding stresses, we can check whether the corresponding combination of stresses indeed leads to a failure. Thus, in the 3-D space $R^{3}$, we have a set $S$ of all possible combinations which lead a failure, and its complement, a set of all combinations which do not lead to a failure.

From the mathematical viewpoint, for every set $S \subseteq R^{3}$, there exist a function $f: R^{3} \rightarrow R$ and a real number $f_{0}$ such that:

- $f(x) \geq f_{0}$ for all the points $x \in S$ and

- $f(x)<f_{0}$ for all $x \notin S$.

For example, as the desired function $f$, we can take a characteristic function of the set $S$, i.e., a function for which $f(x)=1$ for $x \in S$ and $f(x)=0$ for $x \notin S$. For this function, the above separation property occurs for $f_{0}=1$.

In the general case, for an arbitrary set $S$, this function has to be discontinuous. However, for well-behaved sets, we can select this function to be continuous (see, e.g., [5]), and if the boundary is smooth, we can have a smooth function $f(x)$.

Main ideas behind Maxwell's solution. Maxwell's solution is based on two ideas widely used in physics applications:

- on the mathematical idea of ignoring higher order terms in the Taylor expansion, and

- on the physical idea of symmetry.

Ignoring higher order terms in the Taylor expansion: details. In physics, most dependencies are smooth (differentiable). In general, a smooth function $f\left(\sigma_{1}, \sigma_{2}, \sigma_{3}\right)$ can be expanded into Taylor series in $\sigma_{i}$ :

$$
f\left(\sigma_{1}, \sigma_{2}, \sigma_{3}\right)=a_{0}+\sum_{i=1}^{3} a_{i} \cdot \sigma_{i}+\sum_{i=1}^{3} \sum_{j=1}^{3} a_{i j} \cdot \sigma_{i} \cdot \sigma_{j}+\ldots
$$

for appropriate coefficients $a_{0}, a_{i}, a_{i j}, \ldots$

We are interested in ductile materials, i.e., materials that can sustain reasonably large stresses without failing. However, even for the best of such materials, these large stresses are much smaller than the stresses that we can potentially apply. So, we can consider the values $\sigma_{i}$ to be reasonable small and do what physicists usually do - ignore higher order terms in the above expansion.

First try: linear approximation. A natural first approximation is when we ignore quadratic and higher order terms. In this case, we get the following reasonable linear approximation to the desired function $f\left(\sigma_{1}, \sigma_{2}, \sigma_{3}\right)$ :

$$
f\left(\sigma_{1}, \sigma_{2}, \sigma_{3}\right)=a_{0}+\sum_{i=1}^{3} a_{i} \cdot \sigma_{i} .
$$


Due to symmetry, linear approximation leads to average stress. Our main physical idea is to use symmetry. There is nothing special about each direction, hence the coefficients $a_{i}$ corresponding to different directions must be equal: $a_{1}=$ $a_{2}=a_{3}$. Thus, the resulting formula reduced to

$$
f\left(\sigma_{1}, \sigma_{2}, \sigma_{3}\right)=a_{0}+a_{1} \cdot \sum_{i=1}^{3} \sigma_{i}
$$

or, equivalently, to

$$
f\left(\sigma_{1}, \sigma_{2}, \sigma_{3}\right)=a_{0}+3 a_{1} \cdot\left(\frac{1}{3} \cdot \sum_{i=1}^{3} \sigma_{i}\right) .
$$

Thus, the "combined stress" $f$ is proportional to the average stress, and the condition $f \geq f_{0}$ means that the average stress should exceed a certain threshold.

Maxwell's observation: the above linearized solution contradicts to physical symmetry. We have deduced the above solution by using the mathematical idea of symmetry. However, in this situation, there is also a physical symmetry.

Namely, suppose that we have a perfectly spherical body and we apply the exact pressure from all three directions, i.e., we have $\sigma_{1}=\sigma_{2}=\sigma_{3}$. In this case, we have a perfectly symmetric body (invariant with respect to arbitrary rotations around its center) and a perfectly symmetric stress. In a deterministic system, it is thus reasonable to expect that the system will preserve its symmetry.

One can easily see that a fracture is a violation of symmetry. Thus, we can conclude that in this perfectly symmetric case, we should not expect any fractures at all. However, according to our linearized criterion, when $\sigma_{1}=\sigma_{2}=\sigma_{3}$, we have $f=a_{0}+3 a_{1} \cdot \sigma_{1}$, so for sufficiently large stresses, we should have fracture.

Thus, the above linearized solution contradicts to physical symmetry.

Similarly, any kind of irreversible damage on the microlevel is also bound to violate symmetry, so we should not expect the absolutely symmetric stress to cause any damage at all. Thus, we should have $a_{1}=0$, i.e., $a_{1}=a_{2}=a_{3}=0$.

Comment. In practice, fractures do occur even in the symmetric case when all the stresses are equal; however, they occur at a much higher level of stress than when we have different stresses at different directions. So, in the first approximation, we can safely assume that when all three stresses $\sigma_{i}$ are equal, there will be no failure.

From linear to quadratic approximation. We have concluded that due to physical symmetry, there are no linear terms in the Taylor expansion of the function $f: a_{i}=0$. This means that we cannot ignore quadratic terms in the Taylor expansion of the function $f$. A natural next idea is therefore to take quadratic terms into account and to ignore cubic and higher order terms in the expansion of $f$. In this case, we arrive at the following approximate expression for $f$ :

$$
f\left(\sigma_{1}, \sigma_{2}, \sigma_{3}\right)=a_{0}+\sum_{i=1}^{3} \sum_{j=1}^{3} a_{i j} \cdot \sigma_{i} \cdot \sigma_{j} .
$$

Using mathematical symmetry. Due to mathematical symmetry, this expression should not change if we swap two directions (i.e., $1 \leftrightarrow 2$ or $1 \leftrightarrow 3$ ). Because of this invariance requirement:

- all the values $a_{i i}$ should be equal to each other - hence equal to $a_{11}$, and

- all the values $a_{i j}, i \neq j$, should be equal to each otherhence equal to $a_{12}$.

Thus, we conclude that

$$
f\left(\sigma_{1}, \sigma_{2}, \sigma_{2}\right)=a_{0}+a_{11} \cdot \sum_{i=1}^{3} \sigma_{i}^{2}+a_{12} \cdot \sum_{i \neq j} \sigma_{i} \cdot \sigma_{j} .
$$

Using physical symmetry. In the physically symmetric case, when all the stresses coincide $\sigma_{1}=\sigma_{2}=\sigma_{3}=\sigma$, we should not have any combined stress. In this case, the above formula leads to $f=a_{0}+\left(3 a_{11}+6 a_{12}\right) \cdot \sigma^{2}$, so we conclude that $a_{11}=-2 a_{12}$. Thus, $f=a_{0}-a_{12} \cdot V$, where we denoted

$$
\begin{gathered}
V\left(\sigma_{1}, \sigma_{2}, \sigma_{3}\right) \stackrel{\text { def }}{=} 2 \sigma_{1}^{2}+2 \sigma_{2}^{2}+2 \sigma_{3}^{2}-2 \sigma_{1} \cdot \sigma_{2}-2 \sigma_{2} \cdot \sigma_{2}-2 \sigma_{3} \cdot \sigma_{1}= \\
\left(\sigma_{1}-\sigma_{2}\right)^{2}+\left(\sigma_{2}-\sigma_{3}\right)^{2}+\left(\sigma_{3}-\sigma_{1}\right)^{2}
\end{gathered}
$$

Towards the final formula. Since $f$ linearly depends on $V$, the failure condition $f \geq f_{0}$ is equivalent to $V \geq V_{0}$ for an appropriate the threshold $V_{0}$.

The threshold $V_{0}$ can be found out by considering the case of 1-D stress. In this case, e.g., when $\sigma_{1} \neq 0$ and $\sigma_{2}=\sigma_{3}=0$, we know that the failure occurs when $\sigma_{1} \geq \sigma_{f}$. In this case, $V=2 \sigma_{1}^{2}$, so the failure occurs when $V$ reaches the level $V=\sigma_{f}^{2}$. Thus, $V_{0}=2 \sigma_{f}^{2}$.

Final criterion. According to Maxwell's formula, the material fails when $V \geq 2 \sigma_{f}^{2}$, where

$$
V \stackrel{\text { def }}{=}\left(\sigma_{1}-\sigma_{2}\right)^{2}+\left(\sigma_{1}-\sigma_{3}\right)^{3}+\left(\sigma_{2}-\sigma_{3}\right)^{2} .
$$

\section{Maxwell's Mathematical Solution Becomes VON MISES EMPIRICAL FAILURE CRITERION}

In 1913, von Mises experimentally confirmed that for many ductile materials, Maxwell's formula predicts failure well. Because of this confirmation, Maxwell's 1860s mathematical hypothesis is now known as a physically justified empirical fact called von Mises criterion.

According to this criterion, a ductile material fails under the general combination of three stresses $\sigma_{1}, \sigma_{2}$, and $\sigma_{3}$ applied at three orthogonal directions when $V \geq 2 \sigma_{f}^{2}$, where $V \stackrel{\text { def }}{=}$ $\left(\sigma_{1}-\sigma_{2}\right)^{2}+\left(\sigma_{1}-\sigma_{3}\right)^{3}+\left(\sigma_{2}-\sigma_{3}\right)^{2}$.

A similar criterion $V \geq 2 \sigma_{y}^{2}$ can also predict when the yielding starts. For details, see, e.g., [14]. 


\section{NeEd to TAKe Interval UnCERTAinty INTO ACCOUNT}

In real life, we only know the values $\sigma_{i}$ with uncertainty.

Case of interval uncertainty. In some cases, we only know the bounds $\underline{\sigma}_{i}$ and $\bar{\sigma}_{i}$ on the actual (unknown) value of stress. In other words, we only know the interval $\left[\underline{\sigma}_{i}, \bar{\sigma}_{i}\right]$ that contains the actual (unknown) value $\sigma_{i}$.

Main problem: checking whether a material can fail. Different values $\sigma_{i} \in\left[\underline{\sigma}_{i}, \bar{\sigma}_{i}\right]$ lead, in general, to different values of the composite criterion $V$. Since the dependence of $V$ on $\sigma_{i}$ is continuous, in general, possible values of $V$ form an interval $[\underline{V}, \bar{V}]$.

There are three possible situations:

- if $\bar{V}<2 \sigma_{f}^{2}$, this means that all possible value of $V$ are below the failure threshold, so the material will not fail;

- if $\underline{V} \leq 2 \sigma_{f}^{2}<\bar{V}$, this means that the material may fail; on the other hand, it may survive without failure;

- if $2 \sigma_{f}^{2} \leq \underline{V}$, this means that all possible value of $V$ are above the failure threshold, so the material will fail.

In most practical situations, we are interesting in checking whether a material will not fail. To guarantee that the material will not fail, we must check that $\bar{V}<2 \sigma_{f}^{2}$. In other words, it is necessary to find the upper bound $\bar{V}$ of the set of all possible values of $V$ and check whether $\bar{V}<2 \sigma_{f}^{2}$.

In view of this need, in this paper, we will design and analyze algorithms for computing $\bar{V}$.

Comment. In some situations, when we are analyzing the reason for the actual failure, we may want to check whether mechanical failure could have been a reason. In these situations, it is also desirable to compute the lower bound $\underline{V}$.

\section{NeEd to Take FuZZy Uncertainty into Account}

Case of expert knowledge. In some practical situations, we only have expert estimates describing possible stress values. These expert estimates are often described in terms of natural language.

Fuzzy techniques for describing expert uncertainty. In such situations, it is reasonable to use fuzzy sets to formalize the expert knowledge.

From the computational viewpoint, the case of fuzzy uncertainty can be reduced to the case of interval uncertainty. A fuzzy number is uniquely determined by its $\alpha$-cuts. It is known that for every function like $V\left(\sigma_{i}\right)$, for every $\alpha>0$, the $\alpha$-cut for $V$ is equal to the range of $V$ when each $\sigma_{i}$ belongs to the corresponding $\alpha$-cut of $\sigma_{i}$; see, e.g., [1], [6], [10], [11], [12].

Thus, to find the desired degree with which $V \geq 2 \sigma_{f}^{2}$, it is sufficient to compute, for $\alpha=0,0.1,0.2, \ldots, 1$, the upper bounds $\bar{V}$ of the interval for $V$ corresponding to the $\alpha$-cuts. So, from the computational viewpoint, the case of fuzzy uncertainty can be indeed reduced to the case of interval uncertainty.

\section{COMPUTING $V$ UNDER INTERVAL UnCERTAinty: WHAT IS KNOWN}

Computing $V$ is equivalent to computing variance. From the mathematical viewpoint, $V$ is proportional to the sample variance of the observations $\sigma_{i}$.

Computing variance under interval uncertainty: what is known. The problem of computing sample variance under interval uncertainty has been thoroughly analyzed; see, e.g., [8], [9] and references therein.

In particular, it is shown that in general, the problem of computing the corresponding upper bound $\bar{V}$ is computationally difficult (NP-hard). Crudely speaking, NP-hard means that in some cases, we (most probably) have to spend exponential time $\sim c^{n}$ to solve this problem; for exact definitions, see, e.g., [4], [13].

It is also known that the upper bound $\bar{V}$ is always attained when each of the values $\sigma_{i}$ takes one of the extreme values $\underline{\sigma}_{i}$ or $\bar{\sigma}_{i}$; see, e.g., [2], [3].

In other words, to compute $\bar{V}$, it is sufficient to consider $2^{n}$ possible combinations of values $\underline{\sigma}_{i}$ and $\bar{\sigma}_{i}$.

Conclusion for von Mises criterion. For von Mises criterion, the above result means that to compute $\bar{V}$, it is sufficient to consider $2^{3}=8$ possible combinations of values $\underline{\sigma}_{i}$ and $\bar{\sigma}_{i}$.

\section{Can We SpeEd Up Computations?}

The above algorithm requires that we compute the expression $V 8$ times. Each computation of $V$ requires:

- 3 subtractions (to compute $\sigma_{i}-\sigma_{j}$ ),

- 3 multiplications (to compute the squares), and

- 2 additions (to compute $V$ ),

to the total of $3 \cdot 8=24$ multiplications and $(2+3) \cdot 8=40$ additions/subtractions.

Because of the practical importance of this problem, a natural question is: can we compute $\bar{V}$ faster? In this paper, we will show that a speed up is indeed possible.

\section{Can We Speed Up: General Result}

Let us first consider the general problem of estimating variance under interval uncertainty. We will prove that in general, we only need to consider $2^{n}-2$ cases to find the upper bound for the variance, because the maximum is never attained when all the bounds are upper or all the bounds are lower. We also prove that, in general, we cannot pick fewer than $2^{n}-2$ combinations.

In this section, we consider the general case: we have $n$ intervals $\left[\underline{x}_{i}, \bar{x}_{i}\right]$, and we want to compute the range $[\underline{V}, \bar{V}]$ of the population variance

$$
V=\frac{1}{n} \sum_{i=1}^{n}\left(x_{i}-E\right)^{2}
$$

where $x_{i} \in\left[\underline{x}_{i}, \bar{x}_{i}\right]$, where $E \stackrel{\text { def }}{=} \frac{1}{n} \sum_{i=1}^{n} x_{i}$. It was previously known that to compute $\bar{V}$, it is sufficient to compute the value of $V$ for $2^{n}$ possible combinations $\left(x_{1}^{\varepsilon_{1}}, \ldots, x_{n}^{\varepsilon_{n}}\right)$, where $\varepsilon_{i} \in$ 
$\{-,+\}, x_{i}^{+} \stackrel{\text { def }}{=} \bar{x}_{i}$, and $x_{i}^{-\stackrel{\text { def }}{=}} \underline{x}_{i}$. The value $\bar{V}$ is equal to the largest of the resulting $2^{n}$ values $V\left(x_{1}^{\varepsilon_{1}}, \ldots, x_{n}^{\varepsilon_{n}}\right)$.

Proposition 1. For every set of intervals $\left[\underline{x}_{1}, \bar{x}_{1}\right], \ldots,\left[\underline{x}_{n}, \bar{x}_{n}\right]$, the value $\bar{V}$ is equal to the maximum of $2^{n}-2$ values $V\left(x_{1}^{\varepsilon_{1}}, \ldots, x_{n}^{\varepsilon_{n}}\right)$ for all $\left(\varepsilon_{1}, \ldots, \varepsilon_{n}\right)$ for which $\left(\varepsilon_{1}, \ldots, \varepsilon_{n}\right) \neq$ $(+, \ldots,+)$ and $\left(\varepsilon_{1}, \ldots, \varepsilon_{n}\right) \neq(-, \ldots,-)$.

Proposition 2. For every tuple $\left(\varepsilon_{1}, \ldots, \varepsilon_{n}\right)$ for which $\left(\varepsilon_{1}, \ldots, \varepsilon_{n}\right) \neq(+, \ldots,+)$ and $\left(\varepsilon_{1}, \ldots, \varepsilon_{n}\right) \neq(-, \ldots,-)$, there exist $n$ intervals $\left[\underline{x}_{1}, \bar{x}_{1}\right], \ldots,\left[\underline{x}_{n}, \bar{x}_{n}\right]$ for which the maximum $\bar{V}$ is only attained at the given tuple $\left(\varepsilon_{1}, \ldots, \varepsilon_{n}\right)$ and not attained at any other such \pm tuple.

Comment. This result is similar to the ones presented in [7].

Proof of Proposition 1. Let us prove that to compute $\bar{V}$, there is no need to consider the tuple $\left(\bar{x}_{1}, \ldots, \bar{x}_{n}\right)$. If one of the intervals $\left[\underline{x}_{i}, \bar{x}_{i}\right]$ is degenerate, i.e., $\underline{x}_{i}=\bar{x}_{i}$, then this fact is trivially true because this same tuple can also be expressed in a different way, as

$$
\left(\bar{x}_{1}, \ldots, \bar{x}_{i-1}, \underline{x}_{i}, \bar{x}_{i+1}, \ldots, \bar{x}_{n}\right) \text {. }
$$

So, to complete the proof, it is sufficient to consider the case when all the intervals are non-degenerate, i.e., when $\underline{x}_{i}<\bar{x}_{i}$ for all $i$.

Let $i_{0}$ be an index for which $\bar{x}_{i_{0}}$ is the smallest of the $n$ values $\bar{x}_{i}$. Let us show that in this case, replacing $\bar{x}_{i_{0}}$ in the tuple $\left(\bar{x}_{1}, \ldots, \bar{x}_{n}\right)$ with a slightly smaller value $x_{i_{0}}$ will increase $V$ - and thus, the maximum of the variance $V$ cannot be attained at the original all-maxima tuple $\left(\bar{x}_{1}, \ldots, \bar{x}_{n}\right)$.

Let us consider two cases: when all the upper endpoints $\bar{x}_{i}$ are the same and when some are different. If they are all the same, then for the all-maxima tuple, $V=0$. If we replace one of them by a smaller value $x_{i_{0}}<\bar{x}_{i_{0}}$, some values $x_{i}$ will become different and we will get $V>0$.

If some of the values $\bar{x}_{i}$ are different, then some of them are larger than the smallest bound $\bar{x}_{i_{0}}$ and thus, the average $E$ of the upper endpoints is also larger than $x_{i_{0}}=\bar{x}_{i_{0}}: x_{i_{0}}<E$.

It is known that

$$
V=\frac{1}{n} \cdot \sum_{i=1}^{n} x_{i}^{2}-E^{2}
$$

Thus,

$$
\frac{\partial V}{\partial x_{i_{0}}}=\frac{1}{n} \cdot\left(2 x_{i_{0}}-2 E\right)=\frac{2}{n} \cdot\left(x_{i_{0}}-E\right) .
$$

Since $x_{i_{0}}<E$, this derivative is negative, and thus, for slightly smaller values of $x_{i_{0}}<\bar{x}_{i_{0}}$, we will get larger values of $V$. So, in the non-degenerate case, the maximum $\bar{V}$ cannot be attained at an all-maxima tuples.

Similarly, we can prove that to compute $\bar{V}$, there is no need to consider the tuple $\left(\underline{x}_{1}, \ldots, \underline{x}_{n}\right)$. If one of the intervals $\left[\underline{x}_{i}, \bar{x}_{i}\right]$ is degenerate, i.e., $\underline{x}_{i}=\bar{x}_{i}$, then this fact is trivially true because this same tuple can also be expressed in a different way, as

$$
\left(\underline{x}_{1}, \ldots, \underline{x}_{i-1}, \bar{x}_{i}, \underline{x}_{i+1}, \ldots, \underline{x}_{n}\right) .
$$

So, to complete the proof, it is sufficient to consider the case when all the intervals are non-degenerate, i.e., when $\underline{x}_{i}<\bar{x}_{i}$ for all $i$.

Let $i_{0}$ be an index for which $\underline{x}_{i_{0}}$ is the largest of the $n$ values $\underline{x}_{i}$. When all the lower endpoints $\underline{x}_{i}$ are the same, then for the all-minima tuple, $V=0$. If we replace one of them by a larger value $x_{i_{0}}>\underline{x}_{i_{0}}$, some values $x_{i}$ will become different and we will get $V>0$.

If some of the values $\underline{x}_{i}$ are different, then some of them are smaller than the largest bound $\underline{x}_{i_{0}}$ and thus, the average $E$ of the lower endpoints is also smaller than $x_{i_{0}}=\underline{x}_{i_{0}}: E<x_{i_{0}}$. In this case, $\frac{\partial V}{\partial x_{i_{0}}}=\frac{2}{n} \cdot\left(x_{i_{0}}-E\right)>0$. Since $x_{i_{0}}>E$, this derivative is positive, and thus, for slightly larger values of $x_{i_{0}}>\underline{x}_{i_{0}}$, we will get larger values of $V$. The proposition is proven.

Proof of Proposition 2. Let $\left(\varepsilon_{1}, \ldots, \varepsilon_{n}\right)$ be a tuple which is different from $(+, \ldots,+)$ and $(-, \ldots,-)$.

Let us fix some $\delta>0$ (its exact value will be determined later), and let us take $\left[\underline{x}_{i}, \bar{x}_{i}\right]=[-1,-1+\delta]$ when $\varepsilon_{i}=-$ and $\left[\underline{x}_{i}, \bar{x}_{i}\right]=[1-\delta, 1]$ when $\varepsilon_{i}=+$. Since the tuple is different from all pluses, at least of these intervals is negative. For all intervals, $x_{i} \leq 1$, and for at least one negative interval, we have $x_{i} \leq-1+\delta$. Thus, for the average $E=\frac{x_{1}+\ldots+x_{n}}{n}$, we conclude that

$$
E \leq \frac{(n-1) \cdot 1+(-1+\delta)}{n}=\frac{n-2+\delta}{n}=1-\frac{2-\delta}{n} .
$$

If $\frac{2-\delta}{n}>\delta$, i.e., equivalently, if $2-\delta>n \cdot \delta, 2>(n+1) \cdot \delta$ and $\delta<\frac{2}{n+1}$, then we have $E<1-\delta$. Hence, for all $x_{i}$ from the positive intervals, we have $E<x_{i}$.

To guarantee this inequality, let us take $\delta=1 /(n+1)$.

Similarly, since the tuple is different from all pluses, at least of these intervals is positive. For all intervals, $x_{i} \geq-1$, and for at least one positive interval, we have $x_{i} \leq 1-\delta$. Since $\delta<\frac{2}{n+1}$, then we have $E>-1+\delta$. Hence, for all $x_{i}$ from the negative intervals, we have $E>x_{i}$.

From the previous proof, we already know that $\frac{\partial V}{\partial x_{i}}=$ $\frac{2}{n} \cdot\left(x_{i}-E\right)$. For $x_{i}$ from positive intervals, this derivative is positive, so $V$ is strictly increasing and its maximum is attained only when $x_{i}=\bar{x}_{i}$. Similarly, for $x_{i}$ from positive intervals, this derivative is negative, so $V$ is strictly decreasing and its maximum is attained only when $x_{i}=\underline{x}_{i}$. So, the maximum $\bar{V}$ is only attained for the values corresponding to the given tuple. The proposition is proven.

\section{Conclusion fOR THE VON Mises CASE}

In the von Mises case, the above idea reduces the number of values $V$ to compute from 8 to 6 . Thus, we only need $3 \cdot 6=18$ multiplications and $(2+3) \cdot 6=30$ additions/subtractions to compute the upper endpoint $\bar{V}$ corresponding to von Mises criterion. 


\section{Von Mises Case: Possibility of Further Speed UP}

Idea. The possibility speed up comes from the fact that each value $V$ is the sum of 3 terms $\left(\sigma_{i}-\sigma_{j}\right)^{2}$.

For each of these 3 terms, there are only 4 options, corresponding to two choices of $\sigma_{i}=\underline{\sigma}_{i}$ and $\sigma_{i}=\bar{\sigma}_{i}$, and to the two similar choices for $\sigma_{j}$. For each choice, we need one subtraction to compute $\sigma_{i}-\sigma_{j}$ and one multiplication to compute the square. Thus, to compute the values of 4 options for each of these 3 terms, we need $3 \cdot 4=12$ subtractions and $3 \cdot 4=12$ multiplications.

To compute the values of all 6 expressions, we need to add 3 terms. Each computations requires 2 additions, so we need $6 \cdot 12=12$ additions. Thus, overall, we need 12 multiplications and 24 additions/subtractions.

This is almost half of what we needed originally.

\section{Detailed description.}

- First, we compute 4 squares $\left(\underline{\sigma}_{1}-\underline{\sigma}_{2}\right)^{2},\left(\underline{\sigma}_{1}-\bar{\sigma}_{2}\right)^{2}$, $\left(\bar{\sigma}_{1}-\underline{\sigma}_{2}\right)^{2}$, and $\left(\bar{\sigma}_{1}-\bar{\sigma}_{2}\right)^{2}$.

- Then, we compute 4 squares $\left(\underline{\sigma}_{2}-\underline{\sigma}_{3}\right)^{2},\left(\underline{\sigma}_{2}-\bar{\sigma}_{3}\right)^{2}$, $\left(\bar{\sigma}_{2}-\underline{\sigma}_{3}\right)^{2}$, and $\left(\bar{\sigma}_{2}-\bar{\sigma}_{3}\right)^{2}$.

- We compute 4 squares $\left(\underline{\sigma}_{3}-\underline{\sigma}_{1}\right)^{2},\left(\underline{\sigma}_{3}-\bar{\sigma}_{1}\right)^{2},\left(\bar{\sigma}_{3}-\underline{\sigma}_{1}\right)^{2}$, and $\left(\bar{\sigma}_{3}-\bar{\sigma}_{1}\right)^{2}$.

- Finally, we compute the 6 sums

$$
\begin{aligned}
& \left(\underline{\sigma}_{1}-\underline{\sigma}_{2}\right)^{2}+\left(\underline{\sigma}_{2}-\bar{\sigma}_{3}\right)^{2}+\left(\bar{\sigma}_{3}-\underline{\sigma}_{1}\right)^{2} ; \\
& \left(\underline{\sigma}_{1}-\bar{\sigma}_{2}\right)^{2}+\left(\bar{\sigma}_{2}-\underline{\sigma}_{3}\right)^{2}+\left(\underline{\sigma}_{3}-\underline{\sigma}_{1}\right)^{2} ; \\
& \left(\underline{\sigma}_{1}-\bar{\sigma}_{2}\right)^{2}+\left(\bar{\sigma}_{2}-\bar{\sigma}_{3}\right)^{2}+\left(\bar{\sigma}_{3}-\underline{\sigma}_{1}\right)^{2} ; \\
& \left(\bar{\sigma}_{1}-\underline{\sigma}_{2}\right)^{2}+\left(\underline{\sigma}_{2}-\underline{\sigma}_{3}\right)^{2}+\left(\underline{\sigma}_{3}-\bar{\sigma}_{1}\right)^{2} ; \\
& \left(\bar{\sigma}_{1}-\underline{\sigma}_{2}\right)^{2}+\left(\underline{\sigma}_{2}-\bar{\sigma}_{3}\right)^{2}+\left(\bar{\sigma}_{3}-\bar{\sigma}_{1}\right)^{2} ; \\
& \left(\bar{\sigma}_{1}-\bar{\sigma}_{2}\right)^{2}+\left(\bar{\sigma}_{2}-\underline{\sigma}_{3}\right)^{2}+\left(\underline{\sigma}_{3}-\bar{\sigma}_{1}\right)^{2} .
\end{aligned}
$$

- The largest of these 6 sums is the desired value $\bar{V}$.

\section{Additional Speed Up Is Possible for Special SHAPES}

Important practical cases. For a material in general shape, stresses can be of the same size. In practice, we often have a linear or a planar shape. In such cases, stresses in the direction of the shape are usually much larger than in the other directions:

- for a planar shape, we have $\sigma_{3} \ll \sigma_{1}$ and $\sigma_{3} \ll \sigma_{2}$; in precise terms, we have $\bar{\sigma}_{3}<\underline{\sigma}_{1}$ and $\bar{\sigma}_{3}<\underline{\sigma}_{2}$;

- for a linear shape when $\sigma_{2} \ll \sigma_{1}$ and $\sigma_{3} \ll \sigma_{1}$; in precise terms, $\bar{\sigma}_{2}<\frac{1}{2} \cdot \underline{\sigma}_{1}$ and $\bar{\sigma}_{3}<\frac{1}{2} \cdot \underline{\sigma}_{1}$.

Planar case: analysis. Here, $\sigma_{1} \geq \underline{\sigma}_{1}>\bar{\sigma}_{3} \geq \sigma_{3}$ hence $\sigma_{1}>\sigma_{3}$; similarly, $\sigma_{2}>\sigma_{3}$, hence $\sigma_{1}+\sigma_{2}+\sigma_{3}>3 \sigma_{3}$ and $E>\sigma_{3}$. As in the above proofs, we conclude that the maximum is attained when $\sigma_{3}=\underline{\sigma}_{1}$. Due to Proposition 1, for $\bar{V}$, at least one of $\sigma_{1}$ and $\sigma_{2}$ is the lower bound, so we only need to compute $V$ for 3 tuples.
Planar case: algorithm. Here, we compute 3 values $\left(\sigma_{1}-\sigma_{2}\right)^{2}$ (excluding both $\left.\bar{x}_{i}\right), 2$ values $\left(\underline{\sigma}_{3}-\sigma_{1}\right)^{2}$, and 2 values $\left(\underline{\sigma}_{3}-\sigma_{1}\right)^{2}$ - to the total of 7 multiplications and 7 subtractions. After that, we need $3 \cdot 3=6$ additions to compute the needed 3 values of $V$. Overall, we need 7 multiplications and 13 additions/substractions.

Linear case: analysis. In this case, $\sigma_{2}<\sigma_{1}, \sigma_{3}<\sigma_{1}$, hence $E<\sigma_{1}$. Similarly, we have $\sigma_{1}>2 \sigma_{2}$ hence $\sigma_{1}+\sigma_{2}+\sigma_{3}>$ $3 \sigma_{2}$ and $E>\sigma_{2}$ - and similarly $E>\sigma_{2}$. So, maximum is attained for $\bar{\sigma}_{1}, \underline{\sigma}_{2}$, and $\underline{\sigma}_{3}$.

Linear case: algorithm. So, in the linear shape, we only need to compute a single value

$$
V=\left(\bar{\sigma}_{1}-\underline{\sigma}_{2}\right)^{2}+\left(\underline{\sigma}_{2}-\underline{\sigma}_{3}\right)^{3}+\left(\underline{\sigma}_{3}-\bar{\sigma}_{1}\right)^{2},
$$

with 3 multiplications and 5 additions/substractions.

\section{ACKNOWLEDGMENTS}

This work was supported in part by the University Research Institute grant from the University of Texas at El Paso, a Teacher Quality grant from the University of Texas at Austin Charles E. Dana Center and Texas Education Agency, and the Texas Department of Transportation grant No. 0-5453.

The authors are thankful to the anonymous referees for important suggestions.

\section{REFERENCES}

[1] G. Bojadziev and M. Bojadziev, Fuzzy sets, fuzzy logic, applications, World Scientific, Singapore, 1995.

[2] S. Ferson, L. Ginzburg, V. Kreinovich, L. Longpré, and M. Aviles, "Computing Variance for Interval Data is NP-Hard", ACM SIGACT News, 2002, Vol. 33, No. 2, pp. 108-118.

[3] S. Ferson, L. Ginzburg, V. Kreinovich, L. Longpré, and M. Aviles, "Exact Bounds on Finite Populations of Interval Data", Reliable Computing, 2005, Vol. 11, No. 3, pp. 207-233.

[4] M. R. Garey and D. S. Johnson. Computers and Intractability, a Guide to the Theory of NP-Completeness. W. H. Freeman and Company, San Francisco, California, 1979.

[5] J. L. Kelley, General Topology, Springer-Verlag, Berlin-Heidelberg-New York, 1975.

[6] G. Klir and B. Yuan, Fuzzy sets and fuzzy logic, Prentice Hall, New Jersey, 1995.

[7] V. Kreinovich, "Optimal Finite Characterization of Linear Problems with Inexact Data", Reliable Computing, 2005, Vol. 11, No. 6, pp. 479-489.

[8] V. Kreinovich, L. Longpré, S. A. Starks, G. Xiang, J. Beck, R. Kandathi, A. Nayak, S. Ferson, and J. Hajagos, "Interval Versions of Statistical Techniques, with Applications to Environmental Analysis, Bioinformatics, and Privacy in Statistical Databases", Journal of Computational and Applied Mathematics, 2007, Vol. 199, No. 2, pp. 418-423.

[9] V. Kreinovich, G. Xiang, S. A. Starks, L. Longpré, M. Ceberio, R. Araiza, J. Beck, R. Kandathi, A. Nayak, R. Torres, and J. Hajagos, "Towards combining probabilistic and interval uncertainty in engineering calculations: algorithms for computing statistics under interval uncertainty, and their computational complexity", Reliable Computing, 2006, Vol. 12, No. 6, pp. 471-501.

[10] R. E. Moore and W. A. Lodwick, "Interval analysis and fuzzy set theory", Fuzzy Sets and Systems, 2003, Vol. 135, No. 1, pp. 5-9.

[11] H. T. Nguyen and V. Kreinovich, "Nested intervals and sets: concepts, relations to fuzzy sets, and applications", In: R. B. Kearfott and V. Kreinovich (eds), Applications of interval computations, Kluwer, Dordrecht, pp. 245-290.

[12] H. T. Nguyen and E. A. Walker, A First Course in Fuzzy Logic, CRC Press, Boca Raton, Florida, 2006.

[13] C. H. Papadimitriou, Computational Complexity, Addison-Wesley, Reading, Massachusetts, 1994.

[14] J. Vernon, Introduction to Engineering Materials, Palgrave Macmillan, NY, 2003. 